\title{
Análisis de la Competencia Digital Docente: Factor Clave en el Desempeño de Pedagogías Activas con Realidad Aumentada
}

\author{
Analysis of the Digital Teaching Competence: Key Factor in the \\ Performance of Active Pedagogies with Augmented Reality
}

\author{
Arturo Fuentes ${ }^{1}$ \\ Jesús López ${ }^{2 *}$ \\ Santiago Pozo ${ }^{1}$ \\ ${ }^{1}$ Universidad de Granada, España \\ ${ }^{2}$ Universidad Internacional de Valencia, España
}

\begin{abstract}
En una época marcada por los continuos avances tecnológicos, resulta fundamental la formación del profesorado en competencia digital con la finalidad de dar respuesta a las necesidades e inquietudes del alumnado. Los procesos formativos del docente adquieren gran relevancia para disponer de las destrezas requeridas frente a la utilización de nuevos recursos emergentes. Este estudio se centra en conocer la competencia digital docente en la elaboración y aplicación de recursos de realidad aumentada. Se ha seguido un diseño no experimental de tipo descriptivo y correlacional a través de un método cuantitativo. Se han empleado dos cuestionarios, aplicados a una muestra de 2631 docentes. Los resultados obtenidos revelan que el profesorado efectúa una formación continua, realizando de dos a tres cursos al año relacionados con las TIC y otros ámbitos, de manera presencial. Manifiestan destrezas en determinadas áreas de la competencia digital docente como en la seguridad y en la comunicación y colaboración además de un déficit en la creación de contenidos digitales. Profundizando en las áreas más análogas a la realidad aumentada, según en el tipo de centro y etapa educativa, son los centros públicos y de educación primaria los que han revelado mayor nivel competencial.
\end{abstract}

Descriptores: Competencias del docente; Tecnología de la información; Innovación pedagógica; Recursos educacionales.

In a time conditioned by continuous technological advances, it is essential to train teachers in digital competence in order to respond to the needs and concerns of students. The educational processes of the teacher acquire great relevance to have the required skills against the use of new emerging resources. This study focuses on knowing the digital teaching competence in the development and application of augmented reality resources. A non-experimental design of descriptive and correlational type has been followed through a quantitative method. Two questionnaires were used, applied to a sample of 2631 teachers. The results obtained reveal that the teaching staff carries out continuous training, carrying out 2-3 courses per year related to ICT and other areas, in person. They demonstrate skills in certain areas of digital teaching competence, such as security and communication and collaboration, as well as a deficit in the creation of digital content. Deepening in the areas more analogous to augmented reality, according to the type of center and educational stage, it is the public and primary education centers that have revealed the highest level of competence.

Keywords: Teacher qualifications; Information technology; Teaching method innovations; Educational resources.

*Contacto: jesus.lopezb@campusviu.es

ISSN: 1696-4713

www.rinace.net/reice/

revistas.uam.es/reice
Recibido: $\quad 2$ de diciembre 2018

$1^{\text {a }}$ Evaluación: 9 de enero 2019

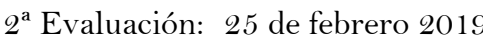

Aceptado: $\quad 3$ de marzo 2019 


\section{Introducción}

La sociedad actual se ha configurado de tal forma que las tecnologías de la información y la comunicación (TIC) ocupan un lugar fundamental (Preston et al., 2015). Sin tener verdadera consciencia de ello, diariamente acudimos a los dispositivos tecnológicos para realizar las tareas más cotidianas. Como consecuencia, cada individuo social se ha visto involucrado de forma obligada a incorporarse a la alfabetización digital para poder desempeñarse con soltura dentro de la era digital (Fuentes y López, 2018).

La población más joven ha sido la que ha vivido dicho proceso de alfabetización con mayor naturalidad, ya que los dispositivos tecnológicos son parte inherente de su realidad (Area, 2015). Estos nativos digitales han asimilado los mecanismos necesarios para el manejo de la tecnología casi de forma instintiva; sin embargo, la población adulta está compuesta fundamentalmente de inmigrantes digitales, ya que estos han necesitado de un considerable empeño para aprender a utilizar dispositivos tecnológicos (Sánchez y Castro, 2013).

Bajo esta perspectiva, resulta fundamental para cualquier sistema educativo que se precie trasladar la realidad tecnológica actual a los espacios de aprendizaje. El deseo de mejorar la calidad de la educación parte de un cambio de dirección en los modelos de escuela (Murillo, 2006) y tiene que constituirse como un fin común para toda la comunidad educativa. La optimización de la educación requiere de compromiso, tenacidad y empeño por parte de los docentes y de las escuelas (Murillo y Krichesky, 2015).

De esta forma, el profesorado se encuentra ante el ambicioso desafío de instruir a su alumnado bajo este nuevo paradigma pedagógico que ha provocado la incorporación de las TIC en los espacios de aprendizaje (Martínez, Hinojo y Rodríguez, 2017). Este hecho se encuentra en consonancia con el incremento de la incorporación de la realidad aumentada (RA) dentro de los espacios educativos (Cabero y Barroso, 2016; Lorenzo y Scagliarini, 2018), aunque su integración en el aula presenta dificultades e inseguridades en los docentes debido a su deficiente nivel de competencia digital y a la escasez de recursos tecnológicos a su alcance (Cózar et al., 2015).

A pesar de que no parece existir una correlación significativa entre la edad o el género de los docentes y su interés por mejorar su nivel de competencia digital (Llorente, 2008; Cózar y Roblizo, 2014), el profesorado no se encuentra lo suficientemente capacitado en el ámbito de las TIC (Fernández, Fernández y Rodríguez, 2018; Fernández y Rodríguez, 2017; Romero et al., 2017).

Por todo lo expuesto, el problema de estudio radica en la necesidad de actualizar las metodologías didácticas mediante la incorporación de las TIC a los espacios formativos, en un ecosistema educativo presidido por una formación docente deficitaria en competencia digital (Passey et al., 2018) y en la utilización de herramientas digitales novedosas como la RA (Rasli et al., 2018). Por ello resulta determinante arrojar luz sobre la situación real de la formación docente en el campo de las TIC y del novedoso recurso de la RA para que la comunidad científica y educativa tomen conciencia del estado de la cuestión. 


\section{Revisión de la literatura}

La enorme influencia que las TIC ejercen en la sociedad actual ha provocado que numerosos docentes hayan considerado necesaria la incorporación de dispositivos tecnológicos a los espacios educativos (Kumar y Kuymar, 2018; McGill, Koppi y Armarego, 2014; Vacek y Rybenska, 2015). Sin embargo, el hecho de que las TIC consigan aportar una verdadera significancia al aprendizaje discente no implica que se nieguen realidades colaterales como la brecha digital y la falta de formación del profesorado (Murillo y Román, 2016). El éxito de incorporar las TIC en los espacios de aprendizaje depende en gran parte de las destrezas del profesorado para organizar el proceso de enseñanza y aprendizaje de forma óptima (Hernández, 2017).

Este hecho genera cierta resistencia al cambio tanto por parte del profesorado como del resto de individuos que componen la comunidad educativa (Sorroza, Jinez, Rodríguez, Caraguay y Sotomayor, 2018) ya que la inmersión de las TIC en la educación requiere de un importante despliegue logístico, formativo y económico. Dentro de las herramientas que las TIC ponen a nuestra disposición se encuentra la ya mencionada augmented reality (Lorenzo y Scagliarini, 2018).

Esta utilidad tecnológica ha vivenciado una rápida y precoz evolución, especialmente durante la última década (Fombona y Pascual, 2017; Prendes, 2015). La RA basa su funcionamiento en la superposición de cualquier tipo de contenido multimedia confeccionado de manera digital pero que se encuentra enlazado con los elementos de la propia realidad del usuario (Ierache et al., 2014). A partir de dispositivos digitales como el smartphone o la tableta electrónica (Cabero, Barroso y Llorente, 2016) se crea un entorno virtual a partir de la información recibida por el entorno real (Cabero y Barroso, 2018; Gómez et al., 2018), produciéndose un enriquecimiento del entorno y de la experiencia del usuario (Ierache et al., 2014; Videla et al., 2017).

Dentro del ámbito educativo, la tecnología basada en la RA está viviendo una enorme proyección debido a la variedad de posibilidades como la dinamización de experiencias de aprendizaje (Chen et al., 2017), la capacidad adaptativa a las preferencias del alumnado actual (Cabero, Barroso y Obrador, 2017), la disponibilidad de un amplio catálogo de aplicaciones (Kamphuis et al., 2014; Toledo y Sánchez, 2017) que lanzan al usuario una ingente cantidad de información multimedia en una gran variedad de formatos (Montecé et al., 2017). Además, este tipo de tecnología tiene el valor añadido de poder adecuarse a cualquier etapa educativa (Garay, Tejada y Castaño, 2017), aumentando la motivación del discente (Videla et al., 2017) gracias a su facilidad de uso y a su carácter interdisciplinar (De la Horra, 2017).

Fombona y Pascual (2017) afirman que todas estas mejoras -anteriormente citadas- que ofrece la RA motivan al docente a demandar formación específica, de manera que pueda elaborar sus propios recursos y utilizarlos en la instrucción de su alumnado. En una sociedad que evoluciona a pasos agigantados, la formación universitaria recibida por los recién titulados en el ámbito de la educación se antoja insuficiente para poder ejercer una docencia actualizada y acorde a las necesidades del alumnado actual (López, Moreno y Pozo, 2018). La formación de los docentes del territorio español ejerce una influencia determinante sobre la calidad de la enseñanza y -por consiguiente- sobre el nivel del alumnado y su grado de consecución de los objetivos formulados para su curso académico (Murillo, 2016). 
En ese ímpetu docente basado en la búsqueda de la calidad y la excelencia educativa y la optimización del proceso de enseñanza y aprendizaje de su alumnado (Murillo y Krichesky, 2015), la implementación de recursos pedagógicos variados y novedosos resulta crucial, especialmente aquellos de índole tecnológica (Murillo, Martínez-Garrido y Hernández-Castilla, 2016). Bajo esta perspectiva, una óptima competencia digital docente se torna como requisito indispensable para poder alcanzar la excelencia en el ejercicio instructivo llevado a cabo por el profesorado actual.

Adentrándonos en la conceptualización de la mencionada competencia digital docente, su origen se encuentra enraizado en la Ponencia de la Competencia Digital Docente que coordina el Instituto Nacional de Tecnologías Educativas y de Formación del Profesorado (INTEF, 2017). En dicha ponencia, se desarrolla el Marco Común De Competencia Digital Docente (Blanco, 2018) en base a las Recomendación del Parlamento Europeo y al Marco estratégico de Educación y Formación (Comisión Europea, 2006, 2009). De esta forma, se produce una estandarización de la competencia digital de los docentes en seis niveles, veintiuna competencias y cinco áreas: Información y Alfabetización informacional, Comunicación y Colaboración, Creación de Contenido Digital, Seguridad y Resolución de problemas (Durán, Gutiérrez y Prendes, 2016; Esteve, 2016).

A pesar de que la mayoría de estudios realizados a nivel nacional que abordan la competencia digital docente no concretiza el nivel alcanzado por el profesorado en cada área, la mayor parte coinciden en la existencia de un escaso número de profesionales con el nivel necesario en competencia digital para poder innovar durante su labor docente (Pérez y Rodríguez, 2016). En una línea similar, autores como Gallego, Gámiz y Gutiérrez (2010), Suárez y otros (2012) y Area y Guarro (2012) entre otros, afirman que a la escasa tecnología actualizada disponible en el aula se le suma la falta de destrezas en competencia digital docente para poder utilizarlas y poder contribuir a la competencia de su alumnado.

Por ello, resulta fundamental la producción de estudios científicos que midan la competencia digital del profesorado y arrojen luz sobre el estado de la cuestión. De esta forma, se podrán tomar medidas urgentes e inmediatas para afrontar con eficacia los nuevos retos que presente la educación del mañana (Carreara y Coiduras, 2012; Centeno y Cubo, 2013).

De esta forma, la presente investigación deriva de la necesidad de conocer la preparación pedagógica de los docentes en materia digital para comprobar si pueden aplicar potencialmente una de las tecnologías emergentes con mayor preponderancia en el panorama educativo actual: la realidad aumentada; una tecnología que requiere de ciertos niveles de destreza en cada una de las áreas de la competencia digital.

En base a todo lo expuesto, el objetivo general que persigue el presente estudio se centra en conocer el nivel de competencia digital que poseen los docentes de las etapas de infantil, primaria y secundaria para la elaboración y puesta en práctica -a nivel pedagógico- de recursos de realidad aumentada.

Para determinar el alcance de este objetivo se han formulado los siguientes objetivos con mayor grado de especificidad:

- Conocer el perfil del profesorado analizado.

- Determinar la frecuencia, ámbito y tipo de formación continua de los docentes. 
- Conocer el nivel de competencia digital necesario para la aplicación de la RA que posee el profesorado en las áreas de la información y alfabetización informacional, comunicación y colaboración, creación de contenidos digitales, seguridad y resolución de problemas.

- Averiguar el grado de asociación entre la etapa educativa de los docentes analizados y su nivel de competencia digital en cuanto a la creación de contenidos digitales relacionados con la RA y la capacidad para la resolución de problemas derivados de su aplicación.

- Determinar la influencia de la etapa educativa en la que imparten docencia los profesionales analizados con respecto al grado demostrado de creación de contenidos digitales de RA y al nivel de resolución de problemas alcanzado.

\section{Método}

\section{Enfoque metodológico}

Con la finalidad de alcanzar los objetivos marcados en este estudio, se lleva a cabo un diseño de investigación no experimental de tipo descriptivo y correlacional bivariado a través de un método de corte cuantitativo (McMillan y Schumacher, 2005).

\section{Variables}

Las variables de estudio que se han tomado en esta investigación parten de los objetivos anteriormente formulados (Hernández, Fernández y Baptista, 2014) y para facilitar su lectura se han establecido las siguientes nomenclaturas:

En la dimensión Sociodemográfica (SD) se encuentran las variables género (GEN), edad (AGE), titulación académica (TITU), tipo de centro educativo (CENT) y etapa educativa (ETAP).

En la dimensión Formación continua (FC) se establecen las variables número de cursos realizados al año (NCUR), tipo de curso (TCUR) y ámbito de formación (ACUR).

En la dimensión Competencia digital docente (CDD) se definen las variables referidas a cada una de las áreas que engloba tal competencia, siendo estas: información y alfabetización informacional (CDINF), comunicación y colaboración (CDCC), creación de contenidos digitales (CDCRE), seguridad (CDSEG) y resolución de problemas (CDRES).

A su vez, todas las variables presentadas abarcan diversos ítems contenidos en el instrumento de recogida de datos con el propósito de obtener un resultado significativo de cada reactivo, permitiendo establecer una valoración de cada variable y, por consiguiente, dimensión.

\section{Muestra y muestreo}

La muestra de estudio abarca un total de 2631 docentes, cuyas características se encuentran recogidas en el cuadro 1. Estos participantes fueron escogidos por medio de un muestreo probabilístico estratificado, tomando en consideración las premisas establecidas por Del Cid, Méndez y Sandoval (2007), Torres, Paz y Salazar (2006). Los estratos muestrales que se definieron para alcanzar la representatividad fueron los siguientes: 1-Género, 2-Edad, 3-Titulación, 4-Tipo de centro y 5-Etapa educativa. 
Cuadro 1. Características sociodemográficas de la muestra

\begin{tabular}{|c|c|c|c|}
\hline VARIABLE & ETIQUETA & ÍTEM & $\mathrm{N}(\%)$ \\
\hline \multirow{2}{*}{ Género } & \multirow{2}{*}{ GEN } & Hombre & $1207(45,88)$ \\
\hline & & Mujer & $1424(54,12)$ \\
\hline \multirow{4}{*}{ Edad } & \multirow{4}{*}{ AGE } & 20-30 años & $485(18,43)$ \\
\hline & & 31-40 años & $712(27,05)$ \\
\hline & & $41-50$ años & $826(31,39)$ \\
\hline & & Más de 50 años & $608(23,11)$ \\
\hline \multirow{4}{*}{ Titulación } & \multirow{4}{*}{ TITU } & Diplomatura & $1063(40,40)$ \\
\hline & & Licenciatura & $1081(41,09)$ \\
\hline & & Grado & $359(13,64)$ \\
\hline & & Doctorado & $128(4,87)$ \\
\hline \multirow{3}{*}{ Tipo de centro } & \multirow{3}{*}{ CENT } & Público & $1266(48,12)$ \\
\hline & & Privado & $655(24,89)$ \\
\hline & & Concertado / Cooperativa & $710(26,99)$ \\
\hline \multirow{3}{*}{ Etapa educativa } & \multirow{3}{*}{ ETAP } & Educación Infantil & $627(23,83)$ \\
\hline & & Educación Primaria & $1174(44,62)$ \\
\hline & & Educación Secundaria & $830(31,55)$ \\
\hline
\end{tabular}

Fuente: Elaboración propia.

Siguiendo las orientaciones postuladas por Hernández, Fernández y Baptista (2014), se establecieron como requisitos para la selección de sujetos de estudio: a) el acceso a la muestra y su aceptación; b) la atención a los requerimientos de la investigación; c) disponibilidad de los participantes para un óptimo y eficaz proceso de recogida de datos.

Asimismo, una vez seleccionados los sujetos de manera aleatoria, para obtener mayores índices de generalización, se utilizó de manera complementaria la técnica por cadena de referencia o bola de nieve, en el que los individuos pertenecientes a cada estrato colaboraron para compartir el instrumento entre sus iguales, estableciendo una cadena de sujetos con la finalidad de conseguir una mayor amplitud del tamaño muestral (Mendieta, 2015).

\section{Instrumentos de obtención de información}

Para efectuar el proceso de recogida de datos se han utilizado dos instrumentos, en concreto dos cuestionarios, siendo uno de ellos de naturaleza ad hoc, para que permitiera satisfacer las necesidades del estudio y diera amparo a los requerimientos investigativos (Alaminos y Castejón, 2006).

El instrumento confeccionado en cuestión integra un total de 35 ítems que han sido catalogados en dos de las tres dimensiones anteriormente citadas (SD-10 ítems y FC-25 ítems). El tipo de respuesta de cada uno de los reactivos presenta un formato variado, encontrándose algunas de tipo Likert con una escala de valoración gradual de 1-4, yendo de más negativo a más positivo (nada-1, poco-2, bastante-3 y totalmente-4) y otras se presentan configuradas mediante elección de respuesta cerrada.

Antes de realizar su función, siguiendo las premisas de Escobar y Cuervo (2008), el cuestionario ha sido sometido a un proceso de validación de contenido por medio de un juicio de expertos conformado por ocho Doctores especialistas en el área de la tecnología educativa de diversas Universidades de España (Granada, Sevilla, Málaga y Córdoba), 
quienes analizaron la herramienta y ofrecieron un feedback con la intención de mejorar su alcance y eficacia.

Una vez optimizado el cuestionario se procedió al análisis de su confiabilidad a través del Alfa $(\alpha)$ de Cronbach para conocer las propiedades psicométricas del instrumento y determinar el nivel de consistencia interna y estabilidad de los datos que se obtengan. La prueba estadística arrojó los siguientes valores: Dimensión SD: $\alpha=0,821$; Dimensión FC: $\alpha=0,857$; permitiendo esclarecer una confiabilidad media en el instrumento de $\alpha=0,839$, cifra que revela una alta consistencia interna y, por consiguiente, elevada fiabilidad del cuestionario al obtener valores resultantes entre el intervalo $0,8<\alpha<1$ (Bisquerra, 2004).

Tras el análisis del instrumento ad hoc, siguiendo las recomendaciones de Corral (2009), se procedió a la realización de una prueba piloto en un contexto similar al presentado que permitiera evitar problemas derivados en su aplicación y reducir los posibles sesgos que pudieran ocasionarse. En concreto, tal test se realizó en un grupo de 30 docentes, tomando una muestra de ensayo de 10 sujetos por cada tipo de centro educativo, cuyos resultados no revelaron problemas de comprensión alguno.

Por otro lado, para la medición de la dimensión CDD se ha utilizado un cuestionario sobre competencias digitales docentes, elaborado por Tourón y otros (2018) en base al marco actual del INTEF (Instituto nacional de tecnologías educativas y de formación del profesorado). Este instrumento ha sido validado por los autores a nivel convergente y discriminante, además de presentar una elevada fiabilidad $(\alpha=0,979)$.

Trabajo de campo

Esta investigación tuvo su origen en el mes de octubre de 2017, estableciéndose un protocolo investigador que quedó marcado en los siguientes procesos:

- Análisis de los centros educativos españoles: Para constituir la muestra de sujetos de estudio, primeramente, se procedió a la consulta de la base de datos propia del Registro estatal de centros educativos no universitarios de España, con la intención de abarcar todo el territorio nacional (https://www.educacion.gob.es/centros/home.do).

- Relaciones burocráticas: Una vez seleccionados los centros educativos, se estableció contacto con los distintos equipos directivos, bajo el propósito de informar acerca de los objetivos del estudio y agradecer su colaboración para transmitir tal información al claustro docente que representan.

- Selección de sujetos y envío de la documentación: Tras el contacto inicial con los órganos colegiados se produjo la confección de la muestra siguiendo los preceptos estipulados en la investigación. Seguidamente, se produjo el envío telemático de los cuestionarios para iniciar la recogida de datos, así como un documento explicativo sobre la confidencialidad y anonimato de la información recabada y su posterior tratamiento analítico.

- Temporalización: Se estableció una duración de 1 mes para el proceso de expansión muestral por parte de los sujetos escogidos de forma aleatoria inicialmente. Una vez transcurrido dicho plazo, se procedió al cierre de recepción de cuestionarios. 
- Tratamiento de la información: Los datos obtenidos fueron tratados a nivel estadístico a través de software específico para optimizar los resultados obtenidos y permitir esclarecer conclusiones pertinentes y de interés para la comunidad científica.

\section{Análisis de datos}

Los datos obtenidos han sido tratados por el programa Statistical Package for the Social Sciences (SPSS) v.22, estableciendo un $p<0,05$ como diferencia estadísticamente significativa, determinado por un nivel de confianza $(Z)$ del $95 \%$ y un margen de error $(e)$ del $5 \%$.

La presentación de los datos se ha realizado mediante estadísticos como la media $\left(\mathrm{M}_{\mathrm{e}}\right)$, la desviación típica (DT), el coeficiente de asimetría de Pearson (CAP) y el coeficiente de apuntamiento de Fisher $\left(\mathrm{CA}_{\mathrm{F}}\right)$. Y para la comparación de variables se ha llevado a cabo la prueba Chi-cuadrado $\left(\chi^{2}\right)$ y v de Cramer ( $\left.V_{\text {Cramer }}\right)$ (Landero y González, 2006).

\section{Resultados}

Los principales hallazgos encontrados en el estudio se detallan a continuación. Comenzando por la formación continua de los docentes analizados (figura 1), según el número de cursos realizados por año académico, una amplia mayoría de estos profesionales $(\mathrm{n}=1803 ; 69 \%)$ efectúa entre dos y tres cursos de formación. En referencia al tipo de curso llevado a cabo en su proceso complementario de reciclaje de conocimientos, más de la mitad del profesorado sigue una modalidad presencial $(n=1424 ; 54 \%)$. Con respecto al ámbito de tal formación, como se visualiza en el diagrama de sectores correspondiente, se produce un reparto cuasi equitativo, destacando una ligera decantación por la temática TIC y otras $(\mathrm{n}=1024 ; 39 \%)$.
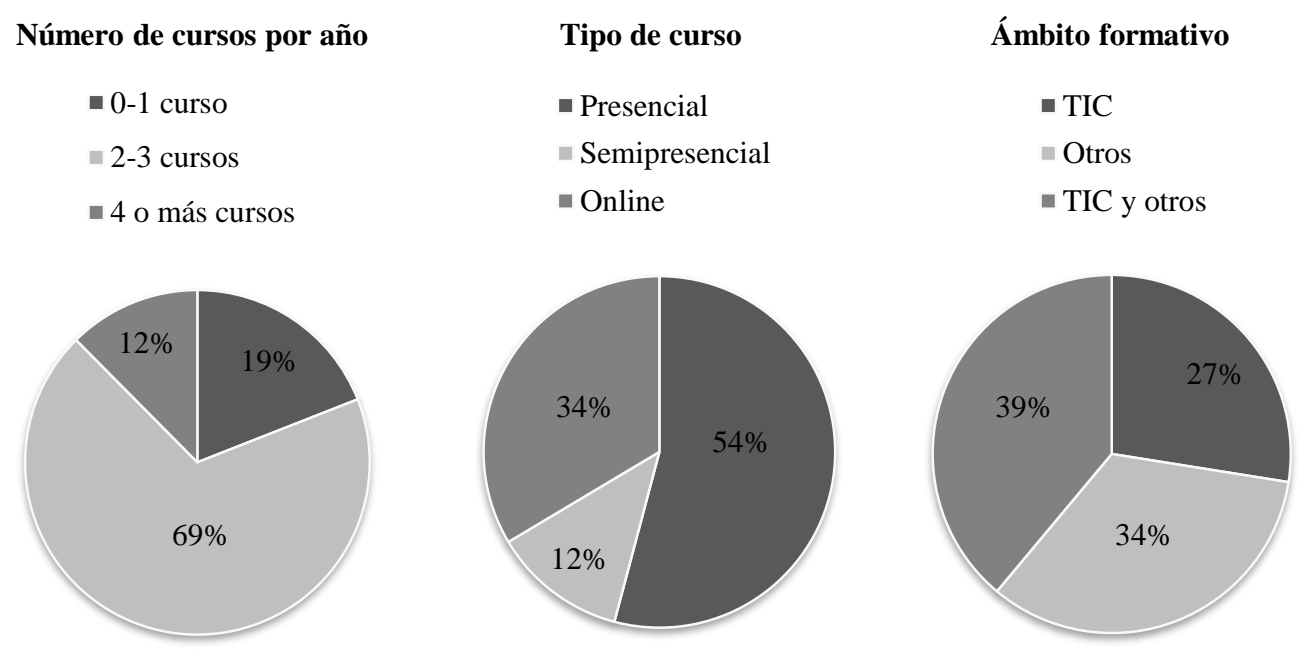

Figura 1. Formación continua del profesorado (NCUR, TCUR y ACUR) Fuente: Elaboración propia.

En el cuadro 2 se detalla el descriptivo con las puntuaciones obtenidas en las distintas áreas que conforman la competencia digital docente de los sujetos encuestados. Como se 
puede apreciar, destacan -por sus elevadas valoraciones por encima del resto- las áreas relativas a la seguridad y a la comunicación y colaboración entre los profesionales de la educación. En cambio, la relacionada con la creación de contenidos digitales conforma el área que más déficit competencial manifiesta disponer el profesorado.

Cuadro 2. Valoraciones de las áreas de la competencia digital docente

\begin{tabular}{lrrrrrrrr}
\hline & \multicolumn{4}{c}{ ESCALA LIKERT $N(\%)$} & \multicolumn{4}{c}{ PARÁMETROS } \\
\cline { 2 - 9 } & \multicolumn{1}{c}{ Nada } & \multicolumn{1}{c}{ Poco } & Bastante & Totalmente & \multicolumn{1}{c}{$\mathbf{M}_{\mathbf{e}}$} & \multicolumn{1}{c}{ DT } & \multicolumn{1}{c}{ CAP } & \multicolumn{1}{c}{ CA $\mathbf{F}$} \\
\hline CDINF & $433(16,46)$ & $784(29,8)$ & $854(32,46)$ & $560(21,28)$ & 2,585 & 0,998 & 1,587 & $-1,053$ \\
CDCC & $255(9,69)$ & $684(25,99)$ & $918(34,89)$ & $774(29,42)$ & 2,841 & 0,957 & 1,922 & $-0,887$ \\
CDCRE & $1134(43,1)$ & $874(33,22)$ & $408(15,51)$ & $215(8,17)$ & 1,887 & 0,948 & 0,935 & $-0,369$ \\
CDSEG & $243(9,24)$ & $337(12,81)$ & $1187(45,12)$ & $864(33,84)$ & 3,015 & 0,908 & 2,218 & $-0,111$ \\
CDRES & $481(18,29)$ & $613(23,3)$ & $779(29,61)$ & $758(28,81)$ & 2,689 & 1,075 & 1,571 & $-1,207$ \\
\hline
\end{tabular}

Nota: $\mathrm{M}_{\mathrm{e}}$ : Media de las puntuaciones. DT: Desviación típica. CAp: Coeficiencia de asimetría de Pearson. $\mathrm{CA}_{\mathrm{F}}$ : Coeficiente de apuntamiento de Fisher.

Fuente: Elaboración propia.

En cuanto al primer análisis correlacional establecido entre el área de creación de contenidos digitales y la resolución de problemas según el tipo de centro educativo, como se muestra en el cuadro 3, ambas variables han alcanzado diferencias estadísticamente significativas, tanto CRCRE $\left(\chi^{2}(6)=74,22, p<0,05\right)$ como CDRES $\left(\chi^{2}(6)=15,35, p<0,05\right)$, ambas con un bajo nivel de dependencia reflejado en los valores de la prueba $\mathrm{V}_{\text {cramer }}<0,25$ (0,238; 0,108; respectivamente). En base a ello, son los centros educativos públicos los que presentan mayor competencia digital en la creación de contenidos digitales y resolución de problemas.

Cuadro 3. Asociación entre CDCRE, CDRES y CENT

\begin{tabular}{|c|c|c|c|c|c|c|c|}
\hline & \multicolumn{3}{|c|}{ TIPO DE CENTRO $N(\%)$} & \multicolumn{4}{|c|}{ PARÁMETROS } \\
\hline & Publico & Privado & Concer./Coop. & $\chi^{2}(g 1)$ & $p$-valor & Cont. & $\mathbf{V}_{\text {Cramer }}$ \\
\hline CDCRE & & & & $74,22(6)$ & 0,001 & 0,166 & 0,238 \\
\hline Nada & $498(8,51)$ & $288(10,95)$ & $348(13,23)$ & & & & \\
\hline Poco & $409(15,54)$ & $224(8,51)$ & $241(9,16)$ & & & & \\
\hline Bastante & $267(10,15)$ & $91(3,46)$ & $50(1,9)$ & & & & \\
\hline Totalmente & $92(3,5)$ & $52(1,98)$ & $71(2,7)$ & & & & \\
\hline CDRES & & & & $15,35(6)$ & 0,018 & 0,076 & 0,108 \\
\hline Nada & $220(8,36)$ & $120(4,56)$ & $141(5,36)$ & & & & \\
\hline Poco & $311(11,82)$ & $158(6,01)$ & $144(5,47)$ & & & & \\
\hline Bastante & $401(15,24)$ & $185(7,03)$ & $193(7,34)$ & & & & \\
\hline Totalmente & $334(12,69)$ & $192(7,3)$ & $232(8,82)$ & & & & \\
\hline
\end{tabular}

Fuente: Elaboración propia.

En esta segunda correlación que se presenta en el cuadro 4, se asocian las variables tomadas de la CDD con la etapa educativa donde se desarrolla profesionalmente el docente. Según los estadísticos efectuados se reflejan diferencias estadísticamente significativas tanto en la variable CDCRE $\left(\chi^{2}(6)=28,68, p=0,002\right)$ como en la CDRES $\left(\chi^{2}(6)=50,21, p=\right.$ $0,001)$ con una escasa intensidad de relación en cuanto a la variable ETAP, según el coeficiente de contingencia. Partiendo de estos resultados, son los docentes de la etapa de Educación Primaria los que muestran mayores índices de competencia digital referida a la creación de contenidos digitales y a la resolución de problemas. 
Cuadro 4. Asociación entre CDCRE, CDRES y ETAP

\begin{tabular}{|c|c|c|c|c|c|c|c|}
\hline & \multicolumn{3}{|c|}{ ETAPA EDUCATIVA $N(\%)$} & \multicolumn{4}{|c|}{ PARÁMETROS } \\
\hline & $\begin{array}{c}\text { Educación } \\
\text { Infantil }\end{array}$ & $\begin{array}{c}\text { Educación } \\
\text { Primaria }\end{array}$ & $\begin{array}{l}\text { Educación } \\
\text { Secundaria }\end{array}$ & $\chi^{q}(\mathrm{gl})$ & $p$-valor & Cont. & $\mathbf{V}_{\text {Cramer }}$ \\
\hline CDCRE & & & & $28,68(6)$ & 0,002 & 0,104 & 0,148 \\
\hline Nada & $279(10,61)$ & $507(19,27)$ & $348(13,23)$ & & & & \\
\hline Poco & $214(8,13)$ & $407(15,47)$ & $253(9,62)$ & & & & \\
\hline Bastante & $97(3,69)$ & $184(6,99)$ & $127(4,83)$ & & & & \\
\hline Totalmente & $37(1,41)$ & $76(2,89)$ & $102(3,88)$ & & & & \\
\hline CDRES & & & & $50,21(6)$ & 0,001 & 0,137 & 0,195 \\
\hline Nada & $108(4,11)$ & $237(9,01)$ & $136(5,17)$ & & & & \\
\hline Poco & $162(6,16)$ & $293(11,14)$ & $158(6,01)$ & & & & \\
\hline Bastante & $191(7,26)$ & $364(13,83)$ & $224(8,51)$ & & & & \\
\hline Totalmente & $166(6,31)$ & $280(10,64)$ & $312(11,86)$ & & & & \\
\hline
\end{tabular}

\section{Discusión y conclusiones}

Los avances producidos a nivel tecnológico en la sociedad de la información y el conocimiento han originado que el profesorado que ejerce la profesión tenga que tomar medidas formativas al respecto, con el fin de satisfacer las necesidades e inquietudes que manifiesta el alumnado, como revelan estudios recientes (Fuentes y López, 2018). Es por ello que esta investigación ha girado en torno al eje formativo del colectivo docente, para conocer su grado de competencia digital al frente de los nuevos recursos y herramientas que proporcionan las TIC en materia educativa.

No se puede someter a comparación el nivel competencial tecnológico alcanzado por los estudiantes con el encontrado en el profesorado analizado en este estudio, por razones evidentes, ya que la incidencia de la estimulación digital ha sido diferente. Los primeros, desde edades tempranas, han asumido la tecnología como un ente cotidiano, familiar y usual, mientras que los segundos -debido a sus reticencias e incertidumbres generadas y formación forzosa- han seguido un desarrollo tardío, como avala la producción científica al respecto (Sánchez y Castro, 2013).

La actualización y renovación de los paradigmas educativos conlleva el compromiso y dedicación de los docentes para asumir el cambio que se está produciendo en las escuelas, como revelan Murillo y Krichesky (2015), siendo necesaria la formación continua del profesorado con la finalidad de asumir el reto de formar a las nuevas generaciones discentes, hecho constatado en estudios previos (Martínez, Hinojo y Rodríguez, 2017) que se encuentran en consonancia con los hallazgos alcanzados en el presente trabajo, cuya formación continua del profesorado es considerada aceptable en base a la temática abarcada y al número de cursos realizados por año. Esto contribuye al fomento de la reducción de la brecha digital existente en este colectivo profesional, como definieron Murillo y Román (2016).

Para integrar con éxito las TIC en educación no solo es necesaria una inversión en equipamientos y recursos sino, además, un despliegue formativo, como revelan Sorroza y otros (2018), cuya afirmación es análoga a los hallazgos de este estudio, en el que el profesorado realiza un ejercicio de actualización en temáticas adyacentes a las TIC. 
A pesar de ello, en congruencia con otras investigaciones (Fernández y Rodríguez, 2017; Fernández, Fernández y Rodríguez, 2018; Romero et al., 2017), tomando de referencia los resultados obtenidos en esta pesquisa, los docentes no disponen de las suficientes destrezas requeridas en alguna de las áreas que articulan a la competencia digital, como se da específicamente en la creación de contenidos digitales, siendo la más deficitaria de todas.

La elaboración de materiales enriquecidos con realidad aumentada exige al profesorado disponer de destrezas a la hora de generar y gestionar este tipo de recursos, así como solventar los posibles problemas y contingencias derivadas de su creación y puesta en práctica, motivo por el cual los docentes demandan y efectúan la formación pertinente, como postulan Fombona y Pascual (2017), no obstante, según los resultados obtenidos, como juicio se determina que no es suficiente para mejorar los índices de competencia digital requeridos.

Tomando en consideración lo expresado por Murillo, Martínez-Garrido y HernándezCastilla (2016) para mejorar y optimizar la calidad de los procesos de enseñanza y aprendizaje es ineludible llevar a cabo prácticas innovadoras que incluyan tecnologías emergentes, motivo por el cual se ha tomado la realidad aumentada en esta investigación.

Si bien la producción científica desarrollada hasta la actualidad en este campo de conocimiento es amplia, tal y como revelan Pérez y Rodríguez (2016), no se han encontrado estudios específicos a nivel nacional donde se ahonde en determinadas áreas a nivel específico, como se ha presentado en este trabajo.

Una vez establecida una comparación entre los hallazgos encontrados en la investigación desplegada con el estado del arte, se concluye que los docentes se preocupan por una formación permanente, realizando para ello entre dos y tres cursos al año, abarcando variedad de temáticas siendo una de ellas las TIC, pero a través de una modalidad tradicional, es decir, de manera presencial. De esta forma, más de la mitad de los docentes analizados (54\%) no aprovechan las potencialidades que brinda la sociedad de la información y el conocimiento, caracterizada por el acceso y la disponibilidad de la información desde cualquier soporte y en cualquier lugar.

A causa de tal proceso formativo de carácter continuo, el profesorado ha reflejado destrezas en las áreas alusivas a la seguridad y a la comunicación y colaboración entre los docentes. No obstante, se hallan destrezas muy carentes en la creación de contenidos, acción que adquiere gran relevancia en el ejercicio docente de una sociedad tecnológica en la que el libro de texto y otros materiales convencionales se están empezando a desplazar para dar cobijo a los nuevos medios didácticos como la realidad aumentada, enriquecidos por los progresos propios de una innovación constante que está incidiendo en la educación.

Asimismo, en esta investigación se ha indagado entre los distintos centros educativos que configuran la oferta educativa en España (públicos, privados y concertados) y las distintas etapas, desde infantil hasta secundaria, en relación al grado de competencia digital en la creación de contenidos y la resolución de problemas, por estar más en consonancia con las destrezas requeridas en la generación y aplicación de materiales didácticos con realidad aumentada. Respecto al tipo de centro y etapa educativa, son los de índole pública y de Educación Primaria los que han revelado mayor nivel competencial en tales áreas. En contraposición a otros estudios de corte similar, la investigación sobre dichas variables contribuye a reforzar un campo de la investigación que se encuentra escaso de literatura, 
permitiendo así la indagación sobre los motivos de incidencia entre el nivel de destrezas digitales del profesorado, el tipo de centro y etapa educativa.

Las prospectivas que surgen de esta investigación se focalizan en la necesidad de fomentar los programas de formación del profesorado en competencia digital para la utilización de tecnologías emergentes -tales como la realidad aumentada- en los espacios educativos, así como dotar a los centros escolares de los recursos requeridos (Cózar et al., 2015) para un despliegue eficiente de metodologías activas y emergentes demandadas por las características tecnológicas de la sociedad actual.

Como limitación encontrada en la realización de este estudio se destaca la escasa colaboración de un número reducido de docentes durante el proceso de recogida de datos. Como futura línea de investigación se propone establecer una comparativa entre dos metodologías emergentes como son la robótica y la realidad aumentada con la finalidad de conocer en cuál de ellas el profesorado dispone de mayor nivel competencial, así como indagar en el motivo de la escasa utilización de la modalidad formativa en línea.

\section{Referencias}

Alaminos, A. y Castejón, J. L. (2006). Elaboración, análisis e interpretación de encuestas, cuestionarios y escalas de opinión. Alicante: Marfil.

Area, M. (2015). La alfabetización digital y la formación de la ciudadanía del siglo XXI. Revista Integra Educativa, 7(3), 21-33.

Area, M. y Guarro, A. (2012). La alfabetización informacional y digital: Fundamentos pedagógicos para la enseñanza y el aprendizaje competente. Revista Española de Documentación Científica, 35, 46-74. https://doi.org/10.3989/redc.2012.mono.977

Bisquerra, R. (2004). Metodología de la investigación educativa. Madrid: La Muralla.

Blanco, S. M. (2018). Marco común de competencia digital docente. Revista Iberoamericana de Educación a Distancia, 21(1), 369-370.

Cabero, J. y Barroso, J. (2016). Ecosistema de aprendizaje con realidad aumentada: Posibilidades educativas. Revista Tecnología, Ciencia y Educación, 5, 141-154.

Cabero, J. y Barroso, J. (2018). Los escenarios tecnológicos en realidad aumentada (RA): Posibilidades Aula Abierta, 47(3), 327-336. https://doi.org/10.17811/rifie.47.3.2018.327-336

Cabero, J., Barroso, J. y Llorente, M. C. (2016). Technology acceptance model \& realidad aumentada: Estudio en desarrollo. Revista Lasallista de Investigación, 13(2), 18-26. https://doi.org/10.22507/rli.v13n2a2

Cabero, J., Barroso, J. y Obrador, M. (2017). Realidad aumentada aplicada a la enseñanza de la medicina. Educación Médica, 1 8(3), 203-208. https://doi.org/10.1016/j.edumed.2016.06.015

Centeno, G. y Cubo, S. (2013). Evaluación de la competencia digital y las actitudes hacia las TIC del alumnado universitario. Revista de Investigación Educativa, 31(2), 517-536. https://doi.org/10.6018/rie.31.2.169271

Chen, P., Liu, X., Cheng, W. y Huang, R. (2017). A review of using augmented reality in education from 2011 to 2016. En J. Popescu (Ed.), Innovations in smart learning (pp. 13-18). Singapur: Springer. https://doi.org/10.1007/978-981-10-2419-1_2 
Comisión Europea. (2006). Recomendación del parlamento europeo y del consejo de 18 de diciembre del 2006 sobre las competencias clave para el aprendizaje permanente. Recuperado de http://infofpe.cea.es/fpe/norm/Rec\%2018_2006.pdf

Comisión Europea. (2009). Marco estratégico educación y formación 2020 (ET2020). Recuperado de http://www.mecd.gob.es/educacion-mecd/mc/redie-eurydice/prioridadeseuropeas/et2020.html

Comisión Europea. (2016). Education and training. Recuperado de https://ec.europa.eu/education/sites/education/files/monitor2016-pt_en.pdf

Corral, Y. (2009). Validez y confiabilidad de los instrumentos de investigación para la recolección de datos. Revista Ciencias de la Educación, 19(33), 228-247.

Cózar, R., Valle, M. D., Hernández, J. A. y Hernández, J. R. (2015). Tecnologías emergentes para la enseñanza de las ciencias sociales: Una experiencia con el uso de realidad aumentada en la formación inicial de maestros. Digital Education Review, 27, 138-153.

Cózar, R. y Roblizo, M. (2014). La competencia digital en la formación de los futuros maestros: Percepciones de los alumnos de los grados de maestro de la facultad de educación de Albacete. Revista Latinoamericana de Tecnología Educativa, 13(2), 119-133.

De la Horra, G. I. (2017). Realidad aumentada, una revolución educativa. Revista de Educación Mediática y TIC, 6(1), 9-22.

Del Cid, A. Méndez, R. y Sandoval, F. (2007). Investigación: Fundamentos y metodología. Ciudad de México: Pearson.

Durán, M., Gutiérrez, I. y Prendes, M. P. (2016). Análisis conceptual de modelos de competencia digital del profesorado universitario. Revista Latinoamericana de Tecnología Educativa, 15(1), 97-114.

Escobar, J. y Cuervo, A. (2008). Validez de contenido y juicio de expertos: Una aproximación a su utilización. Avances en Medición, 6(1), 27-36.

Esteve, F. (2016). Bolonia y las TIC: De la docencia 1.0 al aprendizaje 2.0. La Cuestión Universitaria, $5,58-67$.

Fernández, J., Fernández, M. J. y Rodríguez, J. M. (2018). El proceso de integración y uso pedagógico de las TIC en los centros educativos madrileños. Educación XX1, 21(2), 395416.

Fernández, J. y Rodríguez, A. (2017). TIC y diversidad funcional: Conocimiento del profesorado. European Journal of Investigation in Health, Psychology and Education, 7(3), 157-175. https://doi.org/10.30552/ejihpe.v7i3.204

Fombona, J. y Pascual, M. A. (2017). La producción científica sobre realidad aumentada, un análisis de la situación educativa desde la perspectiva SCOPUS. Revista de Educación Mediática y TIC, $6(1), 39-61$.

Fuentes, A. y López, J. (2018). TIC-TAC: Las tecnologías de la información y la comunicación en la era del acceso. Una experiencia en la educación superior. En del I. Arco y P. Silva (Eds.), Tendencias nacionales e internacionales en organización educativa: Entre la estabilidad y el cambio (pp. 712-727). Madrid: Wolters Kluwer.

Gallego, M. J., Gámiz, V. y Gutiérrez, E. (2010). El futuro docente ante las competencias en el uso de las tecnologías de la información y comunicación para enseñar. Revista de Tecnología Educativa, 34, 1-18. 
Garay, U., Tejada, E. y Castaño, C. (2017). Percepciones del alumnado hacia el aprendizaje mediante objetos educativos enriquecidos con realidad aumentada. Revista de Educación Mediática y TIC, 6(1), 145-164.

Gómez, M., Trujillo, J. M., Aznar, I. y Cáceres, M. P. (2018). Augment reality and virtual reality for the improvement of spatial competences in physical education. Journal of Human Sport and Exercise, 13(2), 189-198.

Hernández, R. M. (2017). Impacto de las TIC en la educación: Retos y perspectivas. Propósitos y Representaciones, 5(1), 325-347. https://doi.org/10.20511/pyr2017.v5n1.149

Hernández, R., Fernández, C. y Baptista, M. P. (2014). Metodología de la investigación. Madrid: McGraw Hill.

Ierache, J., Igarza, S., Mangiarua, N. A., Becerra, M. E., Bevacqua, S. A., Verdicchio, N. N., Ortiz, F. M., Sanz, D., Duarte, N. y Sena, M. (2014). Herramienta de realidad aumentada para facilitar la enseñanza en contextos educativos mediante el uso de las TICs. Revista Latinoamericana de Ingeniería de Software, 2(6), 365-368. https://doi.org/10.18294/relais.2014.365-368

INTEF. (2017). Marco de competencia digital. Madrid: Ministerio de Educación, Ciencia y Deportes.

Kamphuis, C., Barsom, E., Schijven, M. y Christoph, N. (2014). Augmented reality in medical education? Perspectives on Medical Education, 3(4), 300-311. https://doi.org/10.1007/s40037-013-0107-7

Kumar, A. y Kumar, G. (2018). The role of ICT in higher education for the 21 st century: ICT as a change agent for education. Multidisciplinary Higher Education, Research, Dynamics $\Xi^{\circ}$ Concepts: Opportunities \& Challenges for Sustainable Development, 1(1), 76-83.

Landero, R. y González, M. (2006). Estadística con SPSS y metodología de la investigación. Ciudad de México: Trillas.

Llorente, M. (2008). Aspectos fundamentales de la formación del profesorado en TIC. Pixel-Bit. Revista de Medios y Educación, 31, 121-130.

López, J., Moreno, A. y Pozo, S. (2018). Influencia del género y la edad en la formación continua multidisciplinar de los docentes de cooperativas de enseñanza. INNOVA Research Journal, $3(8), 45-59$.

Lorenzo, G. y Scagliarini, C. (2018). Revisión bibliométrica sobre la realidad aumentada en educación. Revista General de Información y Documentación, 28(1), 45-60. https://doi.org/10.5209/RGID.60805

Martínez, L. D., Hinojo, F. J. y Rodríguez, A. M. (2017). Fortalezas, debilidades y concepciones que tienen los profesores al momento de implementar las TIC en sus procesos de enseñanza. Revista Científica Electrónica de Educación y Comunicación en la Sociedad del Conocimiento, $17(2)$, 297-316.

McGill, T., Koppi, T. y Armarego, J. (2014). ICT industry involvement with ICT education and research in universities: Industry perceptions. Innovation in Teaching and Learning in Information and Computer Sciences, 2, 1-18. https://doi.org/10.11120/ital.2014.00010

McMillan, J. H. y Schumacher, S. (2005). Investigación educativa. Madrid: Pearson.

Mendieta, G. (2015). Informantes y muestreo en investigación cualitativa. Investigaciones Andina, $17(30), 1148-1150$.

Montecé, F., Verdesoto, A., Montecé, C. y Caicedo, C. (2017). Impacto de la realidad aumentada en la educación del siglo XXI. European Scientific Journal, 13(25), 129-137. https://doi.org/10.19044/esj.2017.v13n25p129 
Murillo, F. J. (2006). Una dirección escolar para el cambio: Del liderazgo transformacional al liderazgo distribuido. REICE. Revista Iberoamericana sobre Calidad, Eficacia y Cambio en Educación, 4(4), 11-24.

Murillo, F. J. (2016). Hacia un modelo de eficacia escolar. Estudio multinivel sobre los factores de eficacia de las escuelas españolas. REICE. Revista Iberoamericana sobre Calidad, Eficacia y Cambio en Educación, 6(1), 4-28

Murillo, F. J. y Krichesky, G. J. (2015). Mejora de la escuela: Medio siglo de lecciones aprendidas. REICE. Revista Iberoamericana sobre Calidad, Eficacia y Cambio en Educación, 13(1), 69-102.

Murillo, F. J. y Román, M. (2016). Evaluación en el campo educativo: Del sentido a la práctica. Revista Iberoamericana de Evaluación Educativa, 6(1), 7-12.

Murillo, F. J., Martínez-Garrido, C. y Hernández-Castilla, R. (2016). Decálogo para una enseñanza eficaz. REICE. Revista Iberoamericana sobre Calidad, Eficacia y Cambio en Educación, 9(1), 727.

Passey, D., Shonfeld, M., Appleby, L., Judge, M., Saito, T. y Smits, A. (2018). Digital agency: Empowering equity in and through education. Technology, Knowledge and Learning, 23(3), 425-439. https://doi.org/10.1007/s 10758-018-9384-x

Pérez, A. y Rodríguez, M. J. (2016). Evaluación de las competencias digitales autopercibidas del profesorado de educación primaria en Castilla y León (España). Revista de Investigación Educativa, 34(2), 399-415. https://doi.org/10.6018/rie.34.2.215121

Prendes, C. (2015). Realidad aumentada y educación: Análisis de experiencias prácticas. Pixel-Bit. Revista de Medios y Educación, 46, 187-203.

Preston, J. P., Wiebe, S., Gabriel, M., McAuley, A., Campbell, B. y MacDonald, R. (2015). Benefits and challenges of technology in high schools: A voice from educational leaders with a Freire echo. Interchange, 46(2), 169-185. https://doi.org/10.1007/s 10780-015-9240-Z

Rasli, R. M., Zulkefli, N. A. M., Aziz, N. A. A., Razali, F. M., Salim, S. A., Norwawi, N. M. y Basir, N. (2018). A review on teacher training's drawbacks, barriers and challenges: Conventional to digital technology transition. Journal of Fundamental and Applied Sciences, 10(6), 495-511.

Romero, M. R., Castejón, F. J, López, V. M. y Fraile A. (2017). Evaluación formativa, competencias comunicativas y TIC en la formación del profesorado. Comunicar: Revista Científica Iberoamericana de Comunicación y Educación, 25(52), 73-82.

Sánchez, A. y Castro, D. (2013). Cerrando la brecha entre nativos e inmigrantes digitales a través de las competencias informáticas e informacionales. Apertura, 5(2), 6-15.

Sorroza, N. A., Jinez, J. P., Rodríguez, J. E., Caraguay, W. A. y Sotomayor, M. V. (2018). Las TIC y la resistencia al cambio en la educación superior. Revista Científica Mundo de la Investigación y el Conocimiento, 2(2), 477-495.

Suárez, J. M., Almerich, G., Díaz, I. y Fernández, R. (2012). Competencias del profesorado en las TIC. Influencia de factores personales y contextuales. Universitas Psychologica, 11(1), 293309.

Toledo, P. y Sánchez, J. M. (2017). Realidad aumentada en educación primaria: Efectos sobre el aprendizaje. Revista Latinoamericana de Tecnología Educativa, 16(1), 79-92.

Torres, M., Paz, K. y Salazar, F. (2006). Tamaño de una muestra para una investigación de mercado. Boletín Electrónico, 2, 1-13.

Tourón, J., Martín, D., Navarro, E., Pradas, S. y Íñigo, V. (2018). Validación de constructo de un instrumento para medir la competencia digital docente de los profesores. Revista Española de Pedagogía, 76(269), 25-54. https://doi.org/10.22550/REP76-1-2018-02 
Vacek, P. y Rybenska, K. (2015). Research of interest in ICT education among seniors. ProcediaSocial and Behavioral Sciences, 171, 1038-1045. https://doi.org/10.1016/j.sbspro.2015.01.276

Videla, J. J., Sanjuán, A., Martínez, S. y Seoane, A. (2017). Diseño y usabilidad de interfaces para entornos educativos de realidad aumentada. Digital Education Review, 31, 61-79.

\section{Breve CV de los autores}

\section{Arturo Fuentes}

Profesor Ayudante Doctor del Departamento de Métodos de Investigación y Diagnóstico en Educación de la Universidad de Granada. Lleva a cabo su labor investigadora en el Grupo de Investigación AREA HUM-672 (Análisis de la Realidad EducativA) y sus líneas de investigación se centran en los modelos educativos, las tecnologías de acceso a la comunicación en educación y la educación en contextos diferenciados. ORCID ID: https://orcid.org/0000-0003-1970-4895. Email: arturofuentes@ugr.es

\section{Jesús López}

Doctor en Ciencias de la Educación por la Universidad de Granada. Máster en Tecnología Educativa y Competencias Digitales por la UNIR. Ejerce su vocación docente en la Universidad Internacional de Valencia y en el centro educativo concertado Beatriz de Silva (Ceuta, España). Desarrolla su labor investigadora en el Grupo de Investigación AREA HUM-672 (Análisis de la Realidad EducativA) y sus líneas de estudio se centran en la tecnología educativa y en la innovación docente. ORCID ID: https://orcid.org/0000-0003-0823-3370. Email: jesus.lopezb@campusviu.es

\section{Santiago Pozo}

Doctorando en Ciencias de la Educación en la Universidad de Granada. Desarrolla su labor investigadora en el Grupo de Investigación AREA HUM-672 (Análisis de la Realidad EducativA) con especial énfasis en el campo de las tecnologías de la información y la comunicación aplicadas a las metodologías de aprendizaje. Ejerce su vocación docente en el centro educativo concertado Beatriz de Silva (Ceuta, España). Actualmente, se encuentra cursando un Doble Grado en Historia y Filología Hispánica en la Universidad de Burgos. ORCID ID: https://orcid.org/OOOO-0001-8125-4990. Email: santiagopozo@correo.ugr.es 